\title{
SINERGISMO POTENCIAL ENTRE HERBICIDAS INIBIDORES DO FOTOSSISTEMA II E DA SÍNTESE DE CAROTENÓIDES
}

\author{
POTENTIAL SYNERGISM BETWEEN HERBICIDES INHIBITORS OF THE \\ PHOTOSYSTEM II AND CAROTENOIDS SYNTHESIS
}

\section{Nelson Diehl Kruse ${ }^{1}$ Ribas Antonio Vidal $^{2}$ Thomas Trost Bauman ${ }^{3}$ Michelangelo Muzzel Trezzi $^{4}$}

\section{RESUMO}

Herbicidas inibidores do transporte de elétrons na fotossíntese geram estresse oxidativo. Esse estresse normalmente é amenizado pela ação dos carotenóides que dissipam a energia acumulada na forma de calor. Assim, hipotetiza-se que misturas entre herbicidas inibidores do transporte de elétrons, em nível do fotossistema II, e inibidores da síntese de carotenóides possam resultar em sinergismo. $O$ objetivo deste trabalho foi verificar a existência de sinergismo em misturas com herbicidas pertencentes aos dois mecanismos de ação acima mencionados. Para tanto, foram conduzidos dois experimentos: um, com a cultura do milho, nos EUA, em 1998; e outro com cultura da soja, no Brasil, em 1998/99. No experimento com milho, testou-se isoxaflutole nas doses de 0 e 52,5 $\mathrm{ha}^{-1}$ e atrazine nas doses de 0 e $840 \mathrm{~g} \mathrm{ha}^{-1}$, isolados e em mistura. No experimento com soja, testou-se metribuzin, nas doses de 0 e $280 \mathrm{~g} \mathrm{ha}{ }^{-1}$, e clomazone, nas doses de 0 e $560 \mathrm{~g} \mathrm{ha} \mathrm{h}^{-1}$, isolados e em mistura, bem como tratamentos adicionais isolados de metribuzin $560 \mathrm{~g} \mathrm{ha^{-1 }}$ clomazone $1.120 \mathrm{~g} \mathrm{ha}^{-1}$ e testemunha capinada. Na cultura do milho, foram avaliadas as densidades de Abutilon theophrasti (ABUTH), Ipomoea hederacea (IPOHE) e Amaranthus retroflexus (AMARE) aos 52 dias após a aplicação dos herbicidas (DAA) e o rendimento de grãos de milho. Na cultura da soja, foram avaliadas aos 55 DAA, a porcentagem de cobertura e controle de Bidens pilosa (BIDPI), sua densidade aos 30 e 55 DAA, além de massa seca, área foliar e rendimento de grãos de soja. Os resultados indicaram a ocorrência de antagonismo entre os herbicidas, no controle de ABUTH $e$ IPOHE, mas sinergismo entre os mesmos, quando se considera o rendimento de grãos de milho. Para BIDPI, verificou-se sinergismo entre os herbicidas nos percentuais de cobertura $e$ controle, sem valores conclusivos nas demais variáveis. Concluise que, embora seja complexa a comprovação de sinergismo a campo, os resultados indicam, ao menos para uma espécie, a ocorrência de sinergismo na mistura entre inibidores do FSII e da síntese de carotenóides.

Palavras-chave: misturas em tanque, atrazine, clomazone, isoxaflutole, metribuzin.

\section{SUMMARY}

Herbicides that inhibit the electron transport on the photosystem II (PSII) generate oxidative stress. This stress normally is attenuated by carotenoids, which dissipate the electron energy as heat. Hence, we hypothesize that mixtures between PSII inhibitors and carotenoid inhibitors can result in synergism. The objective of this work was to verify the existence of synergism in mixtures of herbicides from both mechanisms of action. Two experiments were conducted in 1998-99 growing season, one with corn, in the USA, and another with soybean in Brazil. At the corn trial, the treatments consisted of isoxaflutole at $52.5 \mathrm{~g} \mathrm{ha}^{-1}$, atrazine at $840 \mathrm{~g} \mathrm{ha}^{-1}$, the mixture of both herbicides, and a weedy control. At the soybean trial, the treatments consisted of metribuzin at $280 \mathrm{~g} \mathrm{ha}^{-1}$, clomazone at $560 \mathrm{~g} \mathrm{ha}^{-1}$, the mixture of both herbicides, with additional isolated treatments of metribuzin at $560 \mathrm{~g} \mathrm{ha}^{-1}$, clomazone at 1,120 $\mathrm{ha}^{-1}$, a weed-free and a weedy control. At the corn crop, assessment included the densities of Abutilon theophrasti (ABUTH), Ipomoea hederacea (IPOHE) and Amaranthus retroflexus (AMARE) at 52 days after the treatments (DAT); and the corn grain yield. At the soybean crop, assessment included Bidens pilosa (BIDPI) density, at 30 and 55 DAT; BIDPI cover, control, dry matter and leaf area, at 55 DAT; and soybean grain yield. Apparent herbicide antagonism occurred at the ABUTH, AMARE and IPOHE control, however, herbicide synergism was observed in the corn grain yield. BIDPI

\footnotetext{
${ }^{1}$ Engenheiro Agrônomo, MSc., Professor Assistente Universidade Federal de Santa Maria, aluno do Curso de Doutorado em Fitotecnia/Universidade Federal do Rio Grande do Sul (UFRGS), bolsista da CAPES/PICDT.

${ }^{2}$ Engenheiro Agrônomo, PhD., Professor, Faculdade de Agronomia, UFRGS, CP 776, 90001-970, Porto Alegre, RS, Brasil. Pesquisador do CNPq. E-mail: vidal@if1.if.ufrgs.br. Autor para correspondência.

${ }^{3}$ Engenheiro Agrônomo, PhD., Professor, Departament of Botany and Plant Pathology, Purdue University, West Lafayette, IN 479061144, USA

${ }^{4}$ Engenheiro Agrônomo, MSc., Professor Assistente CEFET/PR, aluno do Curso de Doutorado em Fitotecnia, UFRGS, bolsista da CAPES/PICDT.
} 
results demonstrated synergism for weed cover and control. Despite the complexity to demonstrate herbicide synergism in the field, the results indicate, at least to one species, the occurrence of synergism in the mixture between PSII inhibitors and carotenoid inhibitors.

Key words: herbicide mixtures, atrazine, isoxaflutole, clomazone, metribuzin.

\section{INTRODUÇÃO}

A utilização simultânea ou seqüencial de dois ou mais herbicidas sobre uma mesma cultura vem sendo cada vez mais empregada na agricultura e representa um avanço nas estratégias de controle sobre as plantas daninhas. Despertam particular interesse as misturas que apresentam sinergismo, pois permitem o uso de doses menores e controlam plantas daninhas resistentes (GRESSEL, 1990). Esse sinergismo, muitas vezes, ocorre quando se misturam dois herbicidas que apresentam diferentes mecanismos de ação, nos quais pode haver uma ação de complementaridade entre os mesmos, com um facilitando a ação física e ou bioquímica do outro (MATTHEWS, 1994).

Os herbicidas inibidores do fotossistema II (FSII), como as triazinas, uréias e uracilas, exercem sua ação fitotóxica ligando-se ao nicho ou "bolso" da proteína $\mathrm{D}_{1}$, no qual a quinona ${ }_{\mathrm{B}}$ deveria ligar-se para receber os elétrons da quinona ${ }_{A}$. Isto bloqueia o transporte de elétrons no fotossistema e gera moléculas de clorofila mais carregadas energeticamente $\left({ }^{3} \mathrm{Chl}\right)$. Nesse estado, elas dão origem a uma reação em cadeia formando radicais livres $\left(\mathrm{R}^{*}\right)$ como: oxigênio singleto $\left({ }^{1} \mathrm{O}_{2}\right)$, superóxido $\left(\mathrm{O}_{2}^{-}\right)$, radical hidroxila $\left(\mathrm{OH}^{*}\right)$ e peróxido de hidrogênio $\left(\mathrm{H}_{2} \mathrm{O}_{2}\right)$, que irão peroxidar os lipídios das membranas, formando novos radicais lipídicos, também capazes de oxidar outros lipídios de membranas, levando assim as plantas tratadas à morte (FUERST \& NORMAN, 1991; GRONWALD, 1994; VIDAL, 1997).

As plantas possuem mecanismos para evitar o estresse oxidativo produzido normalmente em pequeno grau durante a fotossíntese. Entretanto, quando as plantas são tratadas com inibidores do FSII, a quantidade de radicais produzidos ultrapassa a capacidade de destoxificação desses mecanismos. Entre eles estão a ação dos carotenóides, presentes nas membranas dos tilacóides, que protegem contra ${ }^{1} 0_{2},{ }^{3} \mathrm{Chl}$ e peroxidação lipídica, dissipando o excesso de energia da clorofila na forma de calor e a ação do $\alpha$-tocoferol (vitamina $\mathrm{E}$ ), que protege contra a peroxidação lipídica e do $\mathrm{OH}^{*}$, ambos presentes nas membranas do tilacóide (HALL \& RAO, 1980; FUERST \& NORMAN, 1991; SANDMANN et al.,
1991; GRONWALD, 1994; SALISBURY \& ROSS, 1994).

Existem herbicidas que inibem a síntese de carotenóides através da inibição da enzima fitoeno desaturase (FD) como o norflurazon, ou pela inibição da enzima $p$-hidroxifenilpiruvato dioxigenase (HPPD), como o isoxaflutole, ou pela inibição de algum passo ainda não bem esclarecido entre o mevalonato e o geranil-geranil pirofosfato, como o clomazone (SANDMANN et al., 1991; LEE et al., 1997).

No presente trabalho, seguiu-se a hipótese de que a mistura de herbicidas do grupo dos inibidores do FSII com inibidores da síntese de carotenóides pode apresentar sinergismo. Os primeiros geram estresse oxidativo, e os segundos inibem a capacidade da planta de combater esse estresse, tornando-a mais vulnerável e mais facilmente perecível. Assim, o objetivo desse trabalho foi verificar a existência de sinergismo entre duas combinações de herbicidas pertencentes aos dois mecanismos de ação anteriormente citados.

\section{MATERIAIS E MÉTODOS}

Foram conduzidos dois experimentos, a campo, na safra agrícola de 1998-99 em Indiana, EUA, e Rio Grande do Sul, Brasil. Nos EUA, o experimento foi desenvolvido no Centro de Pesquisas Agronômicas da Universidade de Purdue, West Lafayette, USA, com a cultura do milho, cultivada em solo "Haplaquoll" franco argilo-siltoso, no sistema convencional de cultivo (escarificador + enxada rotativa), com $3,5 \%$ de matéria orgânica e pH 6,3. A área encontrava-se em pouso na estação de crescimento anterior. Foi empregada a cultivar Asgrow RX77, semeada em 18 de maio, com espaçamento de $75 \mathrm{~cm}$ entrelinhas, alcançando-se população de 69.000 plantas ha ${ }^{-1}$.

Os tratamentos testados foram organizados num esquema bifatorial, em delineamento de blocos casualizados com cinco repetições. As unidades experimentais mediram três por oito metros. $\mathrm{O}$ primeiro fator foi a aplicação de isoxaflutole (5ciclopropil-4- (2-metilsulfonil-4- trifluorometilbenzoil)isoxazole) nas doses de 0 e $52,5 \mathrm{~g} \mathrm{ha}^{-1}$, e o segundo, a aplicação de atrazine (6-cloro- $N$-etil- $N$ '(1-metiletil)-1,3,5-triazina-2,4-diamina), nas doses de 0 e $840 \mathrm{~g} \mathrm{ha}^{-1}$. Os tratamentos com aplicação de herbicida foram realizados em pré-emergência das plantas cultivada e daninha. Os demais tratos culturais seguiram as recomendações existentes nos EUA para a cultura do milho.

A aplicação dos herbicidas foi realizada em 18/05/98, utilizando-se pulverizador costal 
pressurizado com ar comprimido, munido de barra com seis bicos jato plano tipo "leque" 8003 XLR, espaçados de $50 \mathrm{~cm}$, com consumo de calda equivalente a $280 \ell$ ha $^{-1}$. No momento da aplicação, a temperatura do ar era de $34^{\circ} \mathrm{C}$, e a umidade relativa, de $13 \%$.

As densidades de Abutilon theophrasti (ABUTH), Ipomoea hederacea (IPOHE) e Amaranthus retroflexus (AMARE) foram avaliadas aos 52 dias após aplicação dos herbicidas (DAA), sendo amostradas em área de $16,72 \mathrm{~m}^{2}$ por parcela. $\mathrm{Na}$ mesma área, foi colhido o milho (ZEAMD) em 02/10/98, corrigido o peso para umidade dos grãos de $13 \%$, e expresso em $\mathrm{kg} \mathrm{ha}^{-1}$.

No Brasil, conduziu-se o experimento na Estação Experimental Agronômica da Universidade Federal do Rio Grande do Sul, localizada em Eldorado do Sul, RS, com a cultura da soja, em solo areno-argiloso, contendo $2,4 \%$ de matéria orgânica e $\mathrm{pH}$ 5,5. A cultura foi implantada em sistema de semeadura direta, em sucessão à aveia-preta e ervilhaca, dessecadas em 28/10/98 com glyphosate ( $N$-(fosfonometil) glicina) a $1.080 \mathrm{~g} \mathrm{ha}^{-1}$. A cultivar de soja BR 16 foi semeada em 18/11/98, com espaçamento de $45 \mathrm{~cm}$ entrelinhas e população final média de 241.000 plantas ha $^{-1}$.

Os tratamentos testados também foram organizados em esquema bifatorial (quatro tratamentos), com delineamento de blocos casualizados e quatro repetições. As unidades experimentais mediram dois e meio por oito metros. O primeiro fator foi a aplicação de metribuzin (4amino-6- (1,1-dimetiletil)-3-metiltio-1,2,4-triazin$5(4 \mathrm{H})$-ona) nas doses de 0 e $280 \mathrm{~g} \mathrm{ha}^{-1}$, e o segundo fator, a aplicação de clomazone (2-[(2clorofenil)metil]-4,4-dimetil-3-isoxazolidinona) nas doses de 0 e $560 \mathrm{~g} \mathrm{ha}^{-1}$, com tratamentos adicionais de aplicação de metribuzin na dose de $560 \mathrm{~g} \mathrm{ha}^{-1}$, clomazone na dose de $1.120 \mathrm{~g} \mathrm{ha}^{-1}$, e um tratamento capinado, totalizando sete tratamentos. A aplicação dos herbicidas foram feitas em pré-emergência das plantas cultivada e daninha. Os demais tratos culturais exigidos para o bom desenvolvimento da cultura estiveram de acordo com as recomendações técnicas para a cultura da soja no Rio Grande do Sul e Santa Catarina (EPAGRI-CPPP, 1999).

A aplicação dos herbicidas ocorreu em $18 / 11 / 98$, com pulverizador costal pressurizado a $\mathrm{CO}_{2}$, utilizando-se barra com quatro bicos de jato plano tipo "leque" 8002 XR, espaçados $50 \mathrm{~cm}$, com consumo de calda equivalente a $200 \ell \mathrm{ha}^{-1}$. A temperatura do ar era de $31^{\circ} \mathrm{C}$ e a umidade relativa de $53 \%$. O tratamento capinado sofreu duas capinas, em 15/12/98 e em 05/01/99.
A densidade de Bidens pilosa (BIDPI) foi avaliada aos 33 e 55 DAA, em área de $0,16 \mathrm{~m}^{2}$ por parcela. Os percentuais de cobertura e controle de BIDPI foram obtidos por avaliação visual, considerando-se que $0 \%$ representa nenhuma cobertura e nenhum controle, e $100 \%$, cobertura total do terreno e controle de todas as plantas. A massa seca de BIPDI por $\mathrm{m}^{2}$ foi determinada a partir da coleta de todas as partes aéreas das plantas de BIDPI em área de $0,16 \mathrm{~m}^{2}$, aos 55 DAA, amostrada dentro da área tratada e fora da área útil para colheita da cultura. O material foi seco, até peso constante, em estufa a $60^{\circ} \mathrm{C}$, e pesado. A área foliar de BIDPI foi determinada a partir de cinco plantas coletadas ao acaso, em área contígua à da amostragem de massa seca, aos 55 DAT, medindose a área de todas as folhas, expressas por planta, através de medidor de área foliar LICOR. O rendimento de soja (GLXMA) foi obtido colhendose as duas linhas centrais da parcela, em 21/05/99, numa área de $2,7 \mathrm{~m}^{2}$, corrigindo-se o peso para umidade de $13 \%$, expresso em $\mathrm{kg} \mathrm{ha}^{-1}$.

As densidades de ABUTH, IPOHE e AMARE (transformadas pela transformação logarítmica) e o rendimento de grãos de ZEAMD, foram submetidas à análise da variância em esquema bifatorial seguida por comparação de médias pelo teste $t$, a $5 \%$ de probabilidade de erro. No ensaio com a cultura da soja, a densidade de BIDPI (transformação $\log$ ), os percentuais de cobertura e controle (transformação $\operatorname{arcsen} \sqrt{x+1}$ ), massa seca por $\mathrm{m}^{2}$ e por planta (transformação $\log$ ), área foliar e rendimento de GLXMA foram submetidas à análise da variância e comparação de médias pelo teste $t$, a $5 \%$ de probabilidade de erro. Para avaliar a existência de sinergismo entre os herbicidas, todas variáveis acima foram submetidas à análise segundo o modelo proposto por FLINT et al. (1988), que oferece tratamento estatístico ao método de Colby (COLBY, 1967), através de teste $t$ de significância de contrastes dois por dois. Nesse método, o sinergismo é estimado pelo efeito da interação entre os fatores $\left(\mathrm{I}_{\mathrm{ij}}\right)$, que é calculada subtraindo-se da interação $\left(\mu_{\mathrm{ij}}\right)$, os efeitos dos fatores isolados $\left(\mu_{\mathrm{i} 0} \mathrm{e}\right.$ $\left.\mu_{0 \mathrm{j}}\right)$, e somando-se o efeito dos níveis 0 de cada fator $\left(\mu_{00}\right)\left(\mathrm{I}_{\mathrm{ij}}=\mu_{\mathrm{ij}}-\mu_{\mathrm{i} 0}-\mu_{0 \mathrm{j}}+\mu_{00}\right)$. Haverá a indicação de sinergismo se $\mathrm{I}_{\mathrm{ij}}<0$, e antagonismo se $\mathrm{I}_{\mathrm{ij}}>0$, ambos com significância estatística.

\section{RESULTADOS e DISCUSSÃO}

A densidade de Abutilon theophrasti (ABUTH) sofreu severa redução com os tratamentos de herbicidas, observando-se o melhor resultado com 
a aplicação de isoxaflutole na dose de $52,5 \mathrm{~g} \mathrm{ha}^{-1}$, seguido da mistura de isoxaflutole nessa dose mais atrazine na dose de $840 \mathrm{~g} \mathrm{ha}^{-1}$, e essa superior à aplicação de atrazine isolado, na mesma dose acima (Tabela 1). Comportamento semelhante foi observado também nas densidades de Amaranthus retroflexus (AMARE) e Ipomoea hederacea (IPOHE). No tratamento estatístico do método de Colby (Tabela 1), o valor do efeito estimado positivo e a probalidade de $t$ inferior a $1 \%$ para ABUTH e AMARE apontam para a ocorrência de antagonismo, justamente o oposto da hipótese formulada para a ação conjunta de herbicidas desses dois mecanismos de ação. $O$ efeito antagônico proveniente da aplicação conjunta dos herbicidas pode ser explicado por, pelo menos, três fatores.

Primeiro, com as doses de isoxaflutole empregadas, nas quais os percentuais de controle estavam ao redor de $90 \%$, fica difícil comprovar sinergismo, restando pouca margem para que a mistura comprove sua superioridade. HATZIOS \& PENNER (1985), GREEN (1989) e GRESSEL (1990), referem-se às doses empregadas como fator chave na demonstração de sinergismo. É desejável que as doses escolhidas dos produtos aplicados isoladamente produzam efeito próximo a $50 \%$ de inibição do crescimento, por exemplo, na fase linear

Tabela 1 - Densidade populacional de Abutilon theophrasti (ABUTH), Ipomoea hederacea (IPOHE) e Amaranthus retroflexus (AMARE) aos 52 dias após a aplicação dos herbicidas, rendimento de milho (ZEAMD), efeito estimado da mistura e probabilidade de $t$. West Lafayette, IN, 1998.

\begin{tabular}{|c|c|c|c|c|}
\hline \multirow[t]{2}{*}{ Herbicidas e doses ${ }^{1}$} & \multicolumn{3}{|c|}{ Densidade populacional } & \multirow{2}{*}{$\begin{array}{l}\text { Rendimento de } \\
\text { grãos } \\
\text { ZEAMD }\end{array}$} \\
\hline & ABUTH & AMARE & IPOHE & \\
\hline & \multicolumn{3}{|c|}{ - no de plantas $\mathrm{m}^{-2}$----- } & $\mathrm{Kg} \mathrm{ha}^{-1}$ \\
\hline 1. Infestada & $26,9 \mathrm{a}^{2}$ & $40,4 \mathrm{a}$ & $11,3 \mathrm{a}$ & $6.143 \mathrm{~b}$ \\
\hline 2. isoxaflutole 52,5 & $0,2 \quad \mathrm{~d}$ & $0,6 \mathrm{~b}$ & $2,3 \mathrm{~b}$ & $10.761 \mathrm{a}$ \\
\hline 3. atrazine 840 & $5,0 \mathrm{~b}$ & $0,8 \mathrm{~b}$ & $2,0 \mathrm{bc}$ & $10.449 \mathrm{a}$ \\
\hline 4. isox. $53+$ atraz. $840^{3}$ & $0,6 \mathrm{c}$ & $0,1 \mathrm{~b}$ & $1,6 \mathrm{c}$ & $10.914 \mathrm{a}$ \\
\hline $\mathrm{CV}(\%)^{4}$ & 18 & 33 & 27 & 22 \\
\hline Efeito estimado da mistura & $1,34^{5}$ & 1,35 & 0,71 & $-0,254$ \\
\hline Valor de $t$ & 5,66 & 3,61 & 1,84 & $-2,33$ \\
\hline$t_{\text {calc }}>t_{\text {tab }}$ & 0,0001 & 0,0036 & 0,091 & 0,0382 \\
\hline
\end{tabular}

${ }^{1}$ Doses expressas em $\mathrm{g} \mathrm{ha}^{-1}$.

${ }^{2}$ Médias não seguidas de mesma letra na vertical diferem significativamente pelo teste $t$ a $5 \%$ de probabilidade de erro.

${ }^{3}$ isoxaflutole $52,5+$ atrazine 840

${ }^{4} \mathrm{CV}=$ coeficiente de variação.

${ }^{5}$ Efeito estimado negativo e probabilidade de $\mathrm{t}$ inferior a $5 \%$ indicam sinergismo, e efeito estimado positivo e probabilidade de $\mathrm{t}$ inferior a $5 \%$ indicam antagonismo.

${ }^{6}$ Nível mínimo de significância = probabilidade das diferenças observadas serem devido ao acaso. da curva de resposta. Assim, a mistura dos produtos nessas doses possui ainda espaço para aumento na inibição do crescimento. Se a dose de um ou outro produto estiver próxima do platô superior da curva resposta, não haverá como a mistura produzir resposta. Parece ter sido esse o caso observado nas densidades de ABUTH, IPOHE e AMARE, em que isoxaflutole isolado proporcionou uções de densidade mais intensas do que a em ABUTH, pouco inferiores à mistura em IPOHE e estatisticamente semelhantes em AMARE Segundo, a dose recomendada de isoxaflutole varia entre 75 e $140 \mathrm{~g} \mathrm{ha}^{-1}$, demonstrando que as espécies ABUTH, IPOHE e AMARE foram sensíveis ao isoxaflutole, para permitir controles elevados com dose abaixo da recomendada. Isoxaflutole é inibidor indireto da síntese de carotenóides, já que inibe a enzima HPPD, o que impede a síntese de plastoquinona, da enzima FD, da rota de síntese dos Bloqueia também o transporte de létrons da fotossíntese, ao nível do FSII, pela menor produção da plastoquinona necessária ao sporte de elétrons (LEE et al., 1997). Isto poderia minorar o efeito de atrazine, já que o local de sua ação se encontra neutralizado, em parte, pela ação do isoxaflutole, mascarando um possível efeito cooperativo entre um inibidor de FSII e um inibidor da síntese de carotenóides.

O terceiro fator que pode explicar o aparente antagonismo verificado é que cada espécie pode reagir diferentemente à mistura de herbicidas, podendo haver interações negativas entre a espécie e a absorção, translocação e metabolismo dos componentes da mistura. GREEN (1989) exemplifica isso com a mistura de acifluorfen \{ácido 5-[2cloro-4-(trifluorometil) fenóxi]2-nitrobenzóico\} e bentazon [3-(1-metiletil) - (1H)-2,1,3benzotiadiazina-4(3H)-ona 2,2dióxido] que é sinérgica para Chenopodium album e antagônica em Datura stramonium.

Além desses fatores, YOUNG et $\boldsymbol{a l}$. (1999), em dois anos de trabalho, e em três diferentes tipos de solo, testaram 
sobre ABUTH a dose de $105 \mathrm{~g} \mathrm{ha}^{-1}$ de isoxaflutole isolado e em mistura com atrazine, nas doses de 1.120 e $1.680 \mathrm{~g} \mathrm{ha}^{-1}$, na cultura do milho, e em nenhum caso observaram antagonismo nos percentuais de controle obtido. Pelo contrário, encontraram percentuais de controle para a mistura no mínimo iguais ou superiores aos tratamentos com herbicidas isolados.

Já no rendimento de milho, a mistura de isoxaflutole e atrazine foi sinérgica (Tabela 1), provavelmente pelo melhor controle de IPOHE, que se encontrava em maior densidade no ensaio. Nas demais espécies, ou igualou-se aos tratamentos com os herbicidas isolados no controle de AMARE, ou foi superior ao tratamento com atrazine isolado em ABUTH, e muito próximo de isoxaflutole isolado, embora estatisticamente inferior.

$\mathrm{Na}$ espécie Bidens pilosa (BIDPI), os tratamentos provocaram resultados que demonstram mais diretamente a existência de sinergismo (Tabela 2). A porcentagem de cobertura e de controle de BIDPI, avaliadas aos 55 DAT, revelam que a mistura de metribuzin na dose de $280 \mathrm{~g} \mathrm{ha}^{-1}$ e clomazone na dose de $560 \mathrm{~g} \mathrm{ha}^{-1}$ produziu a menor cobertura vegetal do solo e proporcionou o melhor controle da planta daninha, igualado apenas por metribuzin na dose recomendada de $560 \mathrm{~g} \mathrm{ha}^{-1}$. O efeito estimado da interação (Tabela 2) aponta para a existência de sinergismo da mistura, com efeito estimado negativo e probabilidade inferior a $1 \%$. WESTBERG et al. (1989) em trabalho com mistura de clomazone (280 e $\left.560 \mathrm{~g} \mathrm{ha}^{-1}\right)$, e metribuzin $(200 \mathrm{~g}$ $\mathrm{ha}^{-1}$ ), encontraram sinergismo dos herbicidas no controle de Ipomoea hederacea var. integriuscula em um ano, mas não sendo evidente em outro ano. Em várias espécies estudadas com essa e outras misturas, o sinergismo variou entre as espécies, doses e anos de cultivo.

As densidades de BIDPI apresentaram valores bastante variados (alto coeficiente de variação), sendo que aos 30 DAT não houve diferença entre os tratamentos de herbicidas e a testemunha sem controle. Contudo, aos 55 DAT, apesar dos tratamentos de herbicidas não diferirem entre si, a mistura de herbicidas igualou-se ao tratamento capinado, apresentando a menor densidade de BIDPI. O efeito estimado não apontou significância, sendo os desvios de 0 (aditividade) não significativos (Tabela 2).

A massa seca acumulada de BIPDI reforça o comportamento observado nos percentuais de cobertura e controle, com a mistura apresentando os menores valores, sendo acompanhada apenas pela maior dose de metribuzin, embora o efeito estimado da mistura de herbicidas não tenha apresentado significância aceitável (Tabela 2).

Tabela 2 - Cobertura do solo com Bidens pilosa (BIDPI) e controle aos 55 dias após a aplicação dos herbicidas (DAA), densidade de BIDPI aos aos 30 e 55 DAA, massa seca e área foliar (planta ${ }^{-1}$ ), rendimento de soja (GLXMA), efeito estimado da mistura e probabilidade de t. EEA, UFRGS, Eldorado do Sul, RS, 1998/99.

\begin{tabular}{|c|c|c|c|c|c|c|c|}
\hline \multirow[t]{2}{*}{ Herbicidas e doses ${ }^{1}$} & \multirow{2}{*}{$\begin{array}{l}\text { Cobertura } \\
55 \text { DAT }\end{array}$} & \multirow{2}{*}{$\begin{array}{l}\text { Controle } \\
55 \text { DAT }\end{array}$} & \multicolumn{2}{|c|}{ Densidade de BIDPI } & \multirow{2}{*}{$\begin{array}{l}\text { Massa seca } \\
55 \text { DAT }\end{array}$} & \multirow{2}{*}{$\begin{array}{l}\text { Área foliar } \\
55 \text { DAT }\end{array}$} & \multirow{2}{*}{$\begin{array}{l}\text { Rendimento de } \\
\text { grãos } \\
\text { GLXMA }\end{array}$} \\
\hline & & & 30 DAT & 55 DAT & & & \\
\hline & \multicolumn{2}{|c|}{----------- \% ----------- } & \multicolumn{2}{|c|}{---- $\mathrm{n}^{\mathrm{o}}$ de plantas $\mathrm{m}^{-2}----$} & ---- $(\mathrm{g}) \mathrm{m}^{-2}---$ & $----\mathrm{cm}^{2}----$ & ---- $\mathrm{kg} \mathrm{ha}^{-1}$--- \\
\hline 1.Testemunha Capinada & $0 \quad \mathrm{e}^{2}$ & $100 \mathrm{a}$ & $0 \mathrm{~b}$ & $0 \mathrm{c}$ & $0 \quad \mathrm{e}$ & $0 \quad \mathrm{c}$ & $680 \mathrm{a}$ \\
\hline 2. Testemunha Infestada & $78 \mathrm{a}$ & 0 & $217 \mathrm{a}$ & $317 \mathrm{a}$ & $87,9 \mathrm{a}$ & $265 \mathrm{a}$ & $442 \mathrm{bc}$ \\
\hline 3. metribuzin 280 & $58 \mathrm{~b}$ & $48 \quad \mathrm{~d}$ & $86 a$ & $97 \mathrm{ab}$ & $56,4 \mathrm{ab}$ & $83 \mathrm{~b}$ & $305 \mathrm{c}$ \\
\hline 4. clomazone 560 & $53 \mathrm{~b}$ & 48 & $72 \mathrm{a}$ & $61 \mathrm{ab}$ & $40,9 \mathrm{ab}$ & $53 \mathrm{bc}$ & $467 \mathrm{bc}$ \\
\hline 5. met. $280+$ clom. $560^{3}$ & $11 \mathrm{~d}$ & $84 \mathrm{~b}$ & $53 \mathrm{a}$ & $17 \mathrm{bc}$ & $8,8 \quad \mathrm{~d}$ & $34 \mathrm{bc}$ & $537 \mathrm{ab}$ \\
\hline 6. metribuzin 560 & $20 \mathrm{~cd}$ & $78 \mathrm{bc}$ & $72 \mathrm{a}$ & $63 \mathrm{ab}$ & $16,2 \mathrm{~cd}$ & $75 \mathrm{~b}$ & $433 \mathrm{bc}$ \\
\hline 7. clomazone 1.120 & $24 \mathrm{c}$ & $69 \mathrm{c}$ & $144 \mathrm{a}$ & $116 \mathrm{ab}$ & $30,8 \mathrm{cb}$ & $65 \mathrm{bc}$ & $547 \mathrm{ab}$ \\
\hline $\mathrm{CV}(\%)^{4}$ & 18 & 16 & 39 & 55 & 16 & 56 & 27 \\
\hline Efeito estimado da mistura & $-0,58^{5}$ & $-2,41$ & 1,03 & 0,69 & $-0,43$ & 0,32 & 0,27 \\
\hline Valor de $t$ & $-4,09$ & $-26,11$ & 1,05 & 0,55 & $-1,62$ & 2,25 & 1,47 \\
\hline$t_{\text {calc. }}>t_{\text {tab. }}{ }^{6}$ & 0,003 & 0,0001 & 0,32 & 0,59 & 0,1386 & 0,051 & 0,176 \\
\hline
\end{tabular}

\footnotetext{
${ }^{1}$ Doses expressas em $\mathrm{g} \mathrm{ha}{ }^{-1}$.

${ }^{2}$ Médias não seguidas de mesma letra na vertical não diferem significativamente pelo teste $t$ a $5 \%$ de probabilidade de erro.

3 metribuzin $280+$ clomazone 560 .

${ }^{4} \mathrm{CV}=$ coeficiente de variação, em porcentagem.

${ }^{5}$ Efeito estimado negativo e probabilidade de $\mathrm{t}$ inferior a $5 \%$ indicam sinergismo, e efeito estimado positivo e probabilidade de $\mathrm{t}$ inferior a $5 \%$ indicam antagonismo.

${ }^{6}$ Nível mínimo de significância = probabilidade das diferenças observadas serem devido ao acaso.
} 
A área foliar, avaliada aos 55 DAT, apresentou valores similares entre os tratamentos herbicidas e apontou antagonismo no efeito estimado, com probabilidade próxima a 5\%. Entretanto, observando a média de área foliar, houve tendência da menor média ser obtida pela mistura entre clomazone e metribuzin.

É possível que a dissipação dos produtos no solo tenha afetado o desempenho da mistura a longo prazo. Como metribuzin e clomazone são herbicidas aplicados em pré-emergência, a sua persistência diferencial no solo pode afetar a manifestação do sinergismo nas espécies estudadas. GALLAHER \& MUELLER (1996) obtiveram $22 \mathrm{e}$ 55 dias de meia-vida média para metribuzin e clomazone, respectivamente. Com isso, é possível imaginar-se que 22 dias após a aplicação dos tratamentos a absorção dos produtos por parte das ervas já era proporcionalmente diferente da inicial, fazendo com que o controle obtido aos 55 dias por exemplo, refletisse mais a ação do clomazone que da mistura como um todo. A comparação das densidades de BIPDI aos 30 e 55 DAT nos diferentes tratamentos reforça esse ponto de vista (Tabela 2).

O rendimento de grãos da soja apresentou valores bastante baixos, possivelmente em função do estresse hídrico a que esteve submetida a cultura, principalmente nos estádios vegetativos e início do enchimento de grãos. Apesar disso, a mistura dos herbicidas e a aplicação isolada de clomazone na dose maior $\left(1.120 \mathrm{~g} \mathrm{ha}^{-1}\right)$ não diferiram da testemunha capinada.

Nos dois trabalhos não foram observados sintomas visuais de fitotoxicidade às culturas, indicando que as misturas não potencializaram a ação fitotóxica dos herbicidas, nas doses estudadas. SALZMAN \& RENNER (1992), encontraram sinergismo entre metribuzin e clomazone na injúria sobre a soja provocada por esses herbicidas. Combinações de metribuzin a 140 e $280 \mathrm{~g} \mathrm{ha}^{-1}+$ clomazone a 420 e $840 \mathrm{~g} \mathrm{ha}^{-1}$ provocaram redução da massa seca das hastes de soja, consideradas sinérgicas pelo método de COLBY (1967) e o teste DMS modificado desenvolvido por HAMILL \& PENNER (1973). Embora indesejável do ponto de vista agronômico, esses resultados reforçam a hipótese de sinergismo entre os herbicidas pertencentes aos dois mecanismos de ação.

Atrazine e metribuzin são geradores de estresse oxidativo, que é normalmente combatido por ação dos carotenóides. Isoxaflutole e clomazone são inibidores da síntese de carotenóides e, devem, em tese, quando aplicados em mistura com os anteriores, apresentar efeito sinérgico. Segundo
ZHANG $\boldsymbol{e t}$ al. (1995), as chances de ocorrer interações sinérgicas ou antagônicas em misturas com herbicidas pertencentes a diferentes mecanismos de ação, é a mesma. Apontam ainda para tendência de sinergismo entre herbicidas de famílias químicas similares, enquanto que STREIBIG $\boldsymbol{e} \boldsymbol{t} \boldsymbol{a l}$. (1998) não esperam sinergismo entre herbicidas com modo de ação similar.

\section{CONCLUSÕES}

A comprovação a campo de interação sinérgica mostra-se bastante complexa, seja pelos herbicidas envolvidos, suas doses e persistência, as espécies de plantas daninhas alvo, e a própria metodologia de avaliação.

Em pelo menos uma espécie (Bidens pilosa), a hipótese de sinergismo entre os inibidores do FSII e da síntese de carotenóides é comprovada.

A escolha das doses apropriadas, o número de plantas daninhas presentes na área e sua variabilidade, além das espécies envolvidas na análise, podem afetar a demonstração de sinergismo.

\section{REFERÊNCIAS BIBLIOGRAFICAS}

COLBY, S.R. Calculating synergistic and antagonistic responses of herbicide combinations. Weeds, Columbus, v.15, p.20-22, 1967.

FLINT, J.L., CORNELIUS, P.L., BARRETT, M. Analyzing herbicide interactions: a statistical treatment of Colby's method. Weed Technology, Champaign, v.2, n.3, p.304-309, 1988.

FUERST, E.P., NORMAN, M.A. Interactions of herbicides with photosinthetic electron transport. Weed Science, Champaign, v.39, n.3, p.458-464, 1991.

GALLAHER, K., MUELLER, T.C. Effect of crop presence on persistence of atrazine, metribuzin and clomazone in surface soil. Weed Science, Champaign, v.44, n.3, p.698-703, 1996.

GREEN, J. Herbicide antagonism at the whole plant level. Weed Technology, Champaign, v.3, n.2, p.217-226, 1989.

GRESSEL, J. Synergizing herbicides. Reviews of Weed Science, Champaign, v.5. p.49-82, 1990.

GRONWALD, J.W. Resistance to photosystem II inhibiting herbicides. In: POWLES, S.; HOLTUM, J. (eds.) Herbicide resistance in plants: biology and biochemistry. Boca Raton : CRC, 1994. p.27-60.

EPAGRI-CPPP. Recomendações técnicas para a cultura da soja no Rio Grande do Sul e em Santa Catarina. Chapecó, SC : Epagri-CPPP, 1999. 167p.

HALL, D.O., RAO, K.K. Fotossíntese. São Paulo : EPU/ EDUSP, 1980. 89p.

HAMILL, A.S., PENNER, D. Interaction of alachlor and carbofuran. Weed Science, Champaign, v.21, n.4, p.330-335, 1973.

Ciência Rural, v. 31, n. 4, 2001. 
HATZIOS, K.K., PENNER, D. Interactions of herbicides with other agrochemicals in higher plants. Reviews of Weed Science, Champaign, v.1, p.1-63, 1985.

LEE, D.L., PRISBYLLA, M.P., CROMARTIE, T.H., et al. The discovery and structural requirements of inhibitors of $p$ hydroxyphenylpyruvate dioxygenase. Weed Science, Lawrence, v.45, n.4, p.601-609, 1997.

MATTHEWS, J.M. Management of herbicide resistant weed populations. In: POWLES, S.; HOLTUM, J. (eds.) Herbicide resistance in plants: biology and biochemistry. Boca Raton : CRC, 1994. p.317-335.

SALISBURY, F.B., ROSS, C.W. Fisiologia vegetal. México : Iberoamérica, 1994. 758p.

SALZMAN, F.P., RENNER, K.A. Response of soybean to combinations of clomazone, metribuzin, linuron, alachlor, and atrazine. Weed Technology, Champaign, v.6, n.4, p.922-929, 1992
SANDMANN, G., SCHMIDT, A., LINDEN, H., et al. Phytoene desaturase, the essential target for bleaching herbicides. Weed Science, Champaign, v.39, n.3, p.474-479, 1991.

STREIBIG, J.C. A method for determining the biological effect of herbicide mixtures. Weed Science, Champaign, v.29, n.4, p.469-473, 1981 .

VIDAL, R.A. Herbicidas: mecanismos de ação e resistência de plantas. Porto Alegre : R.A. Vidal, 1997. 165p.

WESTBERG, D.E., OLIVER, L.R., FRANS, R.E. Weed control with clomazone alone and with other herbicides. Weed Technology, Champaign, v.3, n.4, p.678-685, 1989.

YOUNG, B.G., HART, S.E., SIMMONS, F.W. Preemergence weed control in conventional-till corn (Zea mays) with RPA 201772. Weed Technology, Lawrence, v.13. p.471-477, 1999.

ZHANG, J., HAMILL, A.S., WEAVER, S.E. Antagonism and synergism between herbicides: trends from previous studies. Weed Technology, Champaign, v.9, p.86-90, 1995.

Ciência Rural, v. 31, n. 4, 2001. 